\title{
Produtividade e pseudoperfilhamento do alho influenciados pelo nitro- gênio, potássio e cobertura morta
}

\author{
Paulo Espíndola Trani ${ }^{1}$; Mônica Sartori de Camargo²; Dulcinéia Elizabete Foltran²; Rúter Hiroce'; Flávio \\ B Arruda ${ }^{1}$; Haiko Enok Sawazaki ${ }^{1}$ \\ ${ }^{1}$ Instituto Agronômico-APTA, SAA, C. Postal 28, 13012-970 Campinas-SP;. ²APTA, Pólo Centro Sul, UPD Tietê, C. Postal 18, 18530- \\ 000 Tietê-SP; petrani@iac.sp.gov.br; mscamarg@yahoo.com.br
}

\section{RESUMO}

Os alhos nobres vernalizados apresentam tendência ao pseudoperfilhamento, podendo ser influenciados pelo nitrogênio, potássio e cobertura vegetal, mas há poucos estudos sobre o assunto. $\mathrm{O}$ experimento foi realizado em Latossolo Amarelo distrófico, textura média, em Campinas-SP. O delineamento experimental foi em blocos casualizados em esquema fatorial $4 \times 4 \times 2$, composto de quatro doses de $\mathrm{N}\left(0 ; 50 ; 100\right.$ e $\left.150 \mathrm{~kg} \mathrm{ha}^{-1}\right)$, quatro doses de $\mathrm{K}_{2} 0(0$; $50 ; 100$ e $150 \mathrm{~kg} \mathrm{ha}^{-1}$ ), dois sistemas de manejos (com e sem cobertura morta) e três repetições. A área útil de cada parcela foi de 1,5 $\mathrm{m}^{2}$, sendo feito o plantio (maio de 1999 e de 2000) no espaçamento de 0,20 x 0,10 m. Após a colheita (outubro de 1999 e 2000) e a cura (50 dias), foi feita a pesagem, avaliação de pseudoperfilhamento e de outros defeitos, calculando-se as produtividades total e comercial. Houve efeito das doses de nitrogênio, de potássio e da cobertura mortal, de maneira independente sem ocorrer interação entre esses fatores, variando em função de cada avaliação. A adubação nitrogenada aumentou o pseudoperfilhamento e a potássica reduziu esse distúrbio nos dois anos. Os bulbos classificados como miúdos predominaram em 1999 e os médios em 2000. A cobertura vegetal proporcionou menores produções em todas as classes, produtividade total e comercial e não influenciou outros defeitos nos dois anos. A produtividade total máxima estimada foi 3.922 e $8.689 \mathrm{~kg} \mathrm{ha}^{-1}$ com 74 e $107 \mathrm{~kg} \mathrm{ha}^{-1} \mathrm{~N}$ em 1999 e 2000, respectivamente. A produtividade comercial (3.563 $\left.\mathrm{kg} \mathrm{ha}^{-1}\right)$ foi verificada com $68 \mathrm{~kg} \mathrm{ha}^{-1} \mathrm{~N}$ em 1999 e não houve resposta às doses de nitrogênio em 2000. A máxima produção comercial estimada de alho ocorreu com 94,5 kg ha $^{-1} \mathrm{~K}_{2} \mathrm{O}$ em 1999 e, em 2000, a resposta foi linear.

Palavras-chave: adubação, nutrição, Allium sativum, superbrotamento.

\begin{abstract}
Yield and lateral shoot growing of garlic influenced by nitrogen, potassium and mulching
\end{abstract}

Some types of vernalized garlic show tendency to secondary bulb growth, which can be influenced by nitrogen, potassium and mulching, but there are few studies about the subject. The experiment was carried out in a typical Hapludox, medium texture soil in Campinas, São Paulo State, Brazil. The experimental design was in randomized blocks, $4 \times 4 \times 2$ factorial scheme with four rates of $\mathrm{N}$ $\left(0 ; 50 ; 100\right.$ and $\left.150 \mathrm{~kg} \mathrm{ha}^{-1}\right)$, four rates of $\mathrm{K}_{2} 0(0 ; 50 ; 100$ and $150 \mathrm{~kg}$ $\mathrm{ha}^{-1}$ ), two management systems (with and without mulching) and three replications. The useful area of each plot was $1.5 \mathrm{~m}^{2}$ and planting (May, 1999 and 2000) was done in 0.20 x $0.10 \mathrm{~m}$ plots. After harvest (October, 1999 and 2000) and a hardening period (50 days), the bulbs were evaluated for weight, lateral shoot growth and other defects, total yield and commercial production. Nitrogen and potassium rates and mulching influenced independently the garlic characteristics, varying in function of each evaluation. The nitrogen fertilizations increased the lateral shoot growth and potassium reduced this problem in both years. Most of the bulbs were classified as small in 1999 and as medium size in 2000. Mulching decreased yields in all classes and reduced total and commercial yield and did not affect other defects. The estimated maximum total yield was 3,922 and 8,689 $\mathrm{kg} \mathrm{ha}^{-1} \mathrm{~N}$ with 74 and $107 \mathrm{~kg} \mathrm{ha}^{-1} \mathrm{~N}$ in 1999 and 2000 , respectively. The commercial yield $\left(3,563 \mathrm{~kg} \mathrm{ha}^{-1}\right)$ was obtained with $68 \mathrm{~kg} \mathrm{ha}^{-1} \mathrm{~N}$ in 1999. There was no response to $\mathrm{N}$ rates in 2000. The estimated maximum commercial yield was obtained with 94.5 $\mathrm{kg} \mathrm{ha}^{-1} \mathrm{~K}_{2} \mathrm{O} \mathrm{ha}^{-1}$ in 1999 . There was a linear response to rates of potassium in 2000 .

Keywords: fertilization, nutrition, Allium sativum, lateral shoot growth.

(Recebido para publicação em 20 de março de 2007; aceito em 11 de julho de 2008)

$\mathrm{O}$ alho 'Roxo Pérola de Caçador', assim como outras cultivares de alho nobre, pode apresentar um distúrbio denominado pseudoperfilhamento ou superbrotamento ou crescimento secundário, superior aos alhos comuns, depreciando o produto e reduzindo sua produtividade. As suas causas são genético-fisiológicas, sendo caracterizado pela presença de brotação lateral, originada de alongamento das folhas de proteção dos bubilhos, ocorrendo entre as bainhas das folhas normais. Podem ocorrer também grupos de bulbilhos secundários lateralmente aos bulbos. Os pseudocaules das plantas com pseudoperfilhamento são grossos e firmes, mas não suportam o número excessivo de bainhas de folhas adicionais, ocorrendo tombamento prematuro das plantas. Além disso, os bulbos na maturidade são defeituosos (Souza \& Casali, 1986).

Esse distúrbio, por sua vez, é influenciado pelas adubações nitrogenadas e potássicas, que são prá- ticas comuns, uma vez que as extrações desses nutrientes podem alcançar 121,9 e $110,6 \mathrm{~kg} \mathrm{ha}^{-1}$ de $\mathrm{N}$ e $\mathrm{K}_{2} \mathrm{O}$, respectivamente (Silva et al., 1970).

A elevada absorção de nitrogênio é um dos fatores que contribuem de forma marcante para a ocorrência do pseudoperfilhamento, mas os resultados são discrepantes. O aumento do pseudoperfilhamento com a aplicação de nitrogênio já foi mostrado em trabalhos no exterior (Krarup \& Trobok, 1975; Kent et al., 1988) e no Brasil 
Tabela 1. Produtividade total, comercial, pseudoperfilhos, outros defeitos e distribuição nas classes de tamanho 3;4; 5 e 6 do alho 'Roxo Pérola de Caçador' (Total yield, commercial production, lateral shoot growth, other defects and distribution of size classes 3; 4; 5 and 6 of garlic 'Roxo Pérola de Caçador'). Campinas, IAC, 1999-2000.

\begin{tabular}{|c|c|c|c|c|c|c|c|c|}
\hline Cobertura & $\begin{array}{c}\text { Total } \\
\left(\mathbf{k g ~ h a}^{-1}\right)\end{array}$ & $\begin{array}{c}\text { Comercial } \\
\left(\mathrm{kg} \mathrm{ha}^{-1}\right)\end{array}$ & $\begin{array}{c}\text { Classe } 3 \\
(\%)\end{array}$ & $\begin{array}{c}\text { Classe } 4 \\
(\%)\end{array}$ & $\begin{array}{c}\text { Classe } 5 \\
(\%)\end{array}$ & $\begin{array}{c}\text { Classe } 6 \\
(\%)\end{array}$ & $\begin{array}{c}\text { Pseudoperfilhos } \\
(\%)\end{array}$ & $\begin{array}{c}\text { Outros } \\
\text { Defeitos (\%) }\end{array}$ \\
\hline & \multicolumn{8}{|c|}{1999} \\
\hline$\overline{\text { Com }}$ & $3406,90 \mathrm{~b}$ & $2850,94 \mathrm{~b}$ & 50,06 a & $23,29 a$ & $8,08 \mathrm{~b}$ & $1,89 \mathrm{~b}$ & $11,77 b$ & $4,63 a$ \\
\hline Sem & $3875,00 \mathrm{a}$ & 3614,38 a & 49,25 a & 26,44 a & $12,79 \mathrm{a}$ & $4,04 a$ & $3,79 a$ & $3,58 \mathrm{a}$ \\
\hline Média & 3640,95 & 3232,66 & 49,66 & 24,86 & 10,45 & 2,96 & 7,78 & 4,10 \\
\hline $\mathrm{DMS}^{*}$ & 207,88 & 152,28 & 7,72 & 5,40 & 2,77 & 1,82 & 3,27 & 1,88 \\
\hline \multirow[t]{2}{*}{ CV\% } & 6,64 & 15,68 & 20,02 & 22,10 & 21,06 & 20,65 & 21,11 & 20,53 \\
\hline & \multicolumn{8}{|c|}{2000} \\
\hline Com & $7588,40 \mathrm{~b}$ & $6259,40 \mathrm{~b}$ & $13,26 a$ & $24,25 \mathrm{a}$ & $27,93 \mathrm{~b}$ & 18,37 a & 9,66 a & $6,92 \mathrm{a}$ \\
\hline Sem & $8232,20 \mathrm{a}$ & 6984,10 a & 9,96 a & 21,93 a & 33,76 a & 20,19 a & 9,07 a & $5,08 \mathrm{a}$ \\
\hline Média & 7910,3 & 6621,75 & 11,61 & 23,09 & 30,84 & 19,28 & 9,36 & 6,00 \\
\hline $\mathrm{DMS}^{*}$ & 268,05 & 529,56 & 4,88 & 3,32 & 5,33 & 4,80 & 4,01 & 4,88 \\
\hline $\mathrm{CV} \%$ & 11,19 & 9,26 & 21,28 & 23,00 & 21,52 & 19,97 & 22,98 & 20,00 \\
\hline
\end{tabular}

*DMS= Diferença mínima significativa. Médias seguidas da mesma letra na coluna e ano não diferem entre si pelo teste Tukey $5 \%$ (*LSD = Least significant difference. Means followed by the same letter in the column and year did not differ from each other, Tukey’s test, $\mathrm{p}<0.05$ ).

(Alvarenga \& Santos, 1982; Moraes \& Leal, 1986). Dose baixa de N (40 kg ha-1) proporcionou maior produtividade comercial e menor pseudoperfilhamento em um estudo com doses de até $120 \mathrm{~kg}$ ha $^{-1} \mathrm{~N}$ feito por Resende \& Souza (2001). Por outro lado, Seno et al. (1994), estudando doses até $256 \mathrm{~kg} \mathrm{ha}^{-1} \mathrm{~N}$, não observaram efeitos do $\mathrm{N}$ sobre a produtividade comercial e porcentagem de pseudoperfilhamento na cultivar, assim como Silva (1991) para o alho Roxo Pérola de Caçador. Isso mostra a necessidade dos estudos com esse macronutriente.

A adubação potássica tem sido considerada como um possível fator para reduzir o superbrotamento (Büll et al., 2001). Considerando que o nitrogênio e o potássio são os nutrientes mais absorvidos pela cultura, é necessário que o aumento na absorção do nitrogênio seja acompanhado pelo potássio. Caso contrário, o incremento de doses de nitrogênio sem fornecimento de potássio pode acarretar crescimento excessivo das plantas sem a devida sustentação pelo caule, que é promovida com a absorção de K (Marschner, 1995).

Por outro lado, Konkel et al. (1992), estudando doses de $\mathrm{K}_{2} 0(0 ; 40 ; 80$ e 120 $\mathrm{kg} \mathrm{ha}^{-1}$ ), não observaram efeito na produtividade e superbrotamento do alho Juréia, o que foi atribuído ao alto teor inicial desse nutriente no solo. Büll et al. (2002), estudando 4 níveis de $\mathrm{K}_{2} 0$ (4; 8 e $15 \%$ da CTC) e 4 níveis de $\mathrm{N}$ (40; 80;160 e $320 \mathrm{~kg} \mathrm{ha}^{-1}$ ), também não observaram influência das doses de $\mathrm{K}_{2} 0$ na ocorrência desse distúrbio.

Aliado a isso, a cobertura vegetal dos canteiros é uma prática importante para redução das plantas daninhas, manutenção da umidade do solo, podendo proporcionar maior quantidade de cabeças graúdas e médias (Sumi et al., 1986) e maior produtividade do alho (Menezes Sobrinho et al., 1974). Entre os produtores de alho do estado de São Paulo, é comum essa prática, sendo utilizada palha seca de arroz (parte aérea) que é depositada sobre os canteiros.

Quanto ao pseudoperfilhamento, os resultados do uso de cobertura morta são contraditórios. Araújo et al. (1993) verificaram a ocorrência de maior pseudoperfilhamento com a utilização de capim-gordura, palha de arroz comparado ao uso de bagaço de cana. Ao contrário disso, Corrêa et al. (2003), utilizando palha de vegetais para cobertura dos canteiros, obtiveram maior produtividade do alho.

Poucos trabalhos foram realizados sobre o efeito do nitrogênio, potássio e uso de cobertura morta em condições de campo, embora a importância desses fatores no pseudoperfilhamento do alho seja fundamental para reduzir as perdas de qualidade. Avaliou-se a influência da adubação nitrogenada e potássica na produtividade e pseudoperfilhamento do alho "Roxo Pérola de Caçador", sob duas condições de cultivo (com e sem cobertura morta) em dois anos de cultivo.

\section{MATERIAL E MÉTODOS}

Os experimentos foram conduzidos em condições de campo em, CampinasSP em 1999 e 2000. Foi utilizada a cultivar Roxo Pérola de Caçador, um dos principais alhos nobres plantados no Brasil e o de maior expressão comercial no estado de São Paulo.

O delineamento experimental foi em blocos casualizados em esquema fatorial $4 \times 4 \times 2$, composto de doses de $\mathrm{N}$ $\left(0 ; 50 ; 100\right.$ e $\left.150 \mathrm{~kg} \mathrm{ha}^{-1}\right)$ e de $\mathrm{K}_{2} 0(0$; 50; 100 e $150 \mathrm{~kg} \mathrm{ha}^{-1}$ ), dois manejos (com e sem cobertura morta) e três repetições. As doses de $\mathrm{N} \mathrm{e} \mathrm{K}_{2} 0$ foram parceladas aos 30 e 50 dias após a brotação do alho. As parcelas constituíram-se de canteiros de 3,0 x 1,0 m, sendo a área útil 1,5 $\mathrm{m}^{2}$.

O Latossolo Amarelo distrófico (Embrapa, 1999) utilizado apresentou as características: areia grossa $=330 \mathrm{~g} \mathrm{~kg}^{-1}$; areia fina $=170 \mathrm{~g} \mathrm{~kg}^{-1}$; silte $=110 \mathrm{~g} \mathrm{~kg}^{-1}$ e argila $=390 \mathrm{~g} \mathrm{~kg}^{-1}$. A análise química do solo inicial revelou: matéria orgâni$\mathrm{ca}=21,5 \mathrm{~g} \mathrm{dm}^{-3} ; \mathrm{P}$ (resina) $=20 \mathrm{mg} \mathrm{dm}^{-3}$; $\mathrm{K}, \mathrm{Ca}$ e $\mathrm{Mg}=1,2 ; 19$ e $1,1 \mathrm{mmol}_{\mathrm{c}} \mathrm{dm}^{-3}$, respectivamente; $\mathrm{H}+\mathrm{Al}=28 \mathrm{mmol}_{\mathrm{c}} \mathrm{dm}^{-3}$; $\mathrm{pH}\left(\mathrm{CaCl}_{2}\right)=5,3 ; \mathrm{CTC}=59,7 \mathrm{mmol}_{\mathrm{c}}^{\mathrm{c}} \mathrm{dm}^{-3}$; $\mathrm{V} \%=53$, conforme metodologia utilizada pelo Instituto Agronômico de Campinas (Raij et al., 1997).

Aplicou-se $2 \mathrm{t} \mathrm{ha}^{-1}$ de calcário dolomítico fino (90\% de PRNT) aos 30 dias antes do plantio do alho. A adubação de plantio em todas as parcelas foi 


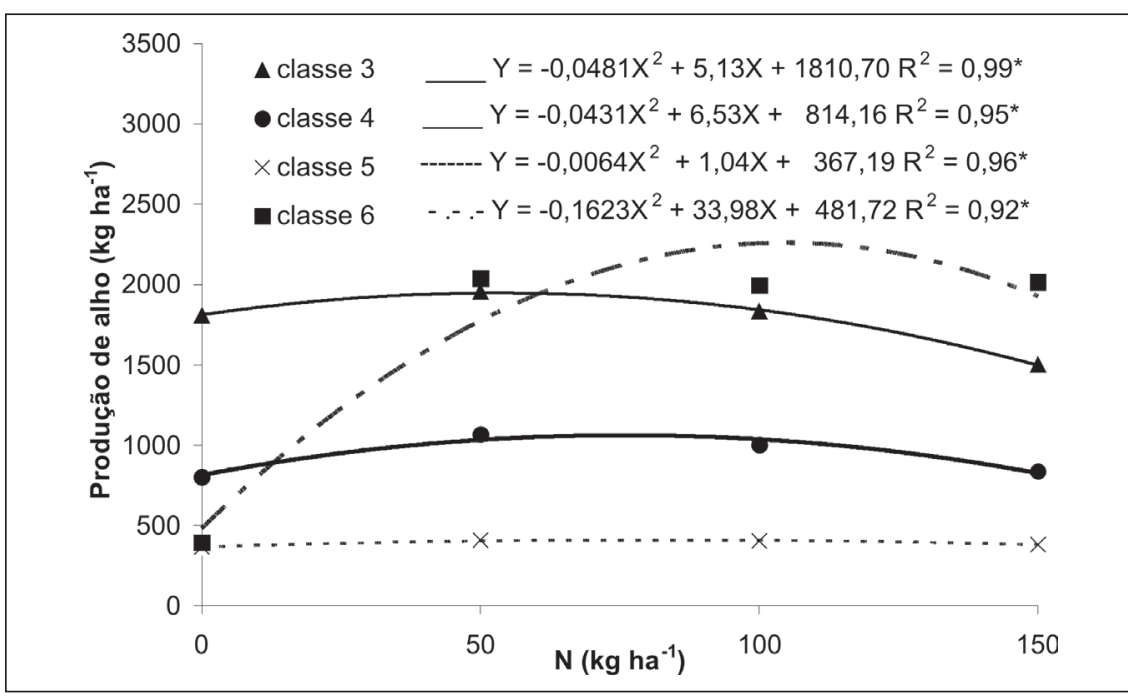

Figura 1. Produção das classes de bulbos de alho no ano de 2000 em função das doses de $\mathrm{N}$ $(* \mathrm{P}<0,05)$ (Production in classes of garlic's bulbs in year 2000 as affected by $\mathrm{N}$ rates $(* \mathrm{p}<0,05))$. Campinas, IAC, 1999-2000.

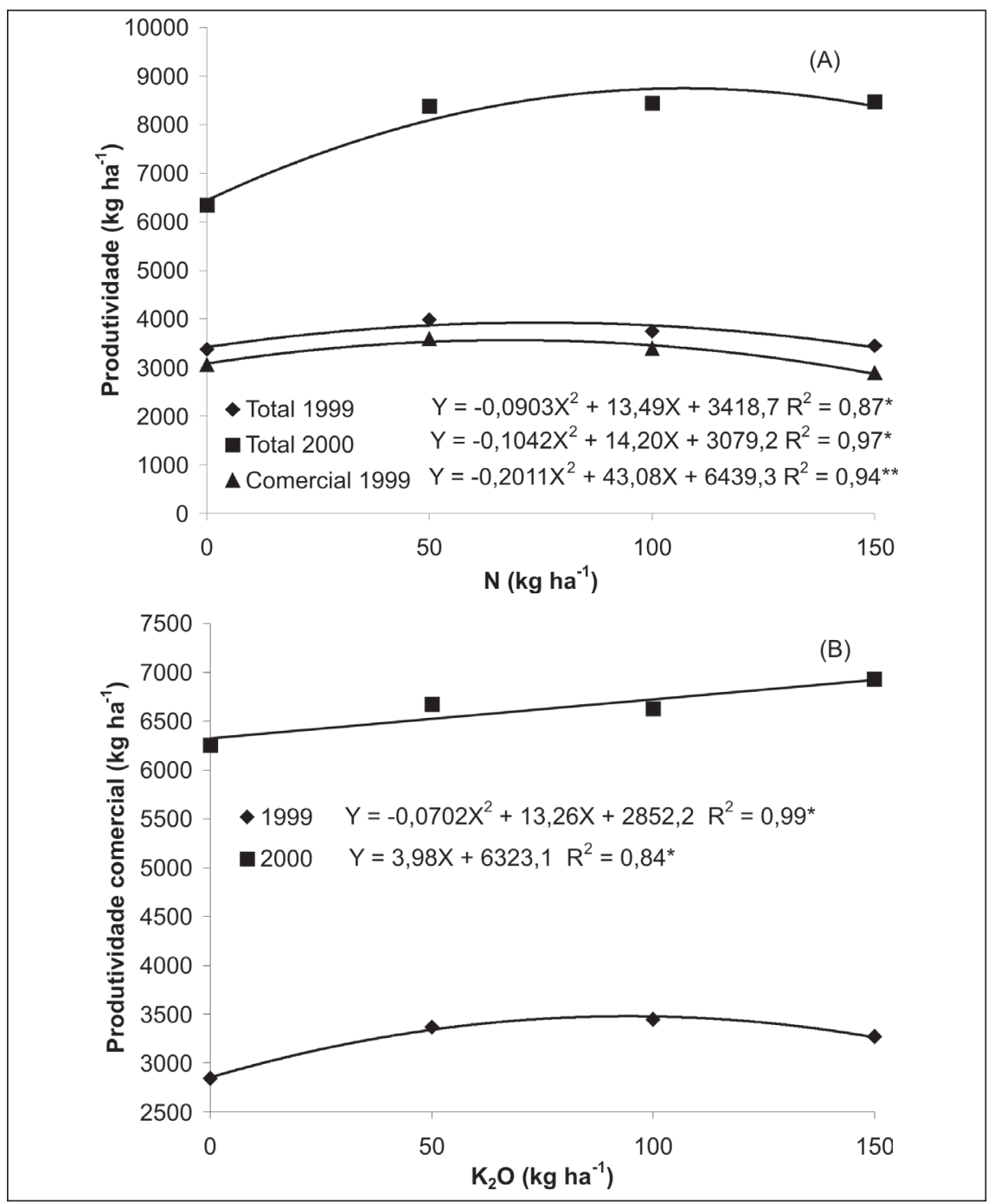

Figura 2. Produtividade total e comercial do alho em função das doses de $\mathrm{N}$ (A) e de $\mathrm{K}_{2} 0$ (B). $\left({ }^{*} \mathrm{p}<0,05\right)$ (Total yield and commercial yield of garlic as affected by $\mathrm{N}$ rates (A) and $\mathrm{K}_{2} \mathrm{O}$ rates (B). $\left.(* \mathrm{p}<0,05)\right)$. Campinas, IAC, 1999-2000. feita 10 dias antes do plantio com $30 \mathrm{~kg}$ $\mathrm{ha}^{-1} \mathrm{~N}, 360 \mathrm{~kg} \mathrm{ha}^{-1} \mathrm{P}_{2} \mathrm{O}_{5}, 3 \mathrm{~kg} \mathrm{ha}^{-1} \mathrm{~B}, 3$ $\mathrm{kg} \mathrm{ha}^{-1} \mathrm{Zn}$ e $1,5 \mathrm{~kg} \mathrm{ha}^{-1} \mathrm{Mn}$, conforme Trani et al. (1997). Durante o ciclo da cultura foram feitas duas pulverizações com oxicloreto de cobre nos dois anos.

A cobertura morta aplicada logo após o plantio do alho constituiu-se de palha de arroz (parte aérea seca). $\mathrm{O}$ alho 'Roxo Pérola de Caçador' foi vernalizado a $5^{\circ} \mathrm{C}$ por 40 dias. O plantio dos bulbilhos nas parcelas foi realizado no início de maio de 1999 e 2000, no espaçamento de 0,20 entre linhas e 0,10 $\mathrm{m}$ entre plantas. Os tratos culturais e fitossanitários foram feitos segundo Tavares et al. (1998).

Os bulbos foram colhidos da área útil de cada parcela em outubro de 1999 e 2000. Após 50 dias de cura, procedeuse à pesagem dos bulbos com pseudoperfilhamento (PS) e com outros defeitos (OD), calculando-se a produtividade total de bulbos (PT) e a produção comercial (PC=PT-OD-PS). Os alhos foram classificados conforme o diâmetro transversal dos bulbos, segundo portaria do MAPA de 1992, citada por Resende. (2004): a) classe 3= miúdo $(32-37 \mathrm{~mm})$; b) classe $4=$ pequeno (38-42 mm); c) classe 5= médio (43-47 $\mathrm{mm})$; d) classe $6=$ grande $(48-56 \mathrm{~mm})$; e) classe $7=$ graúdo $(>56 \mathrm{~mm})$.

$\mathrm{O}$ teste $\mathrm{F}$ para análise de variância foi aplicado para as variáveis analisadas, sendo, posteriormente, utilizado o teste de Tukey para avaliação das diferenças entre médias de cobertura vegetal e a análise de regressão polinomial para as doses de $\mathrm{N} \mathrm{e}_{2} 0$, segundo Nogueira (1999) com o auxílio do programa SAS (SAS Institute, 1996).

\section{RESULTADOS E DISCUSSÃO}

A cobertura vegetal do solo e as doses de nitrogênio e potássio apresentaram efeitos significativos independentes, variando de acordo com as características do alho avaliadas nos dois anos de estudo.

A produtividade média total de alho em 1999 foi baixa comparada à média nacional $\left(6,4 \mathrm{t} \mathrm{ha}^{-1}\right)$ e de 2000 (Tabela 1). Isso pode ser atribuído ao menor tamanho dos bulbilhos utilizados em 1999 com peso médio de 2 gramas (peneira 
3) em relação àqueles de 2000 com peso médio de 3 gramas (peneira 2). De acordo com Soares (1979), maiores produtividades são obtidas com bulbilhos maiores. Aliado a isso, a baixa produção em 1999 também pode estar relacionada à possível alta carga de vírus do alho utilizado no plantio, que pode reduzir de forma acentuada sua produtividade. Essa grande quantidade de vírus nos bulbilhos ocorre geralmente, devido ao excesso de multiplicações feitas por alguns pequenos produtores ao longo dos anos. Os valores observados em 1999, no entanto, não são tão inferiores à média (Tavares et al., 1998) do estado de São Paulo (6,4 t ha' ${ }^{-1}$, podendo o pseudoperfilhamento ter sido proveniente de causas genéticas e não apenas por problema fisiológico com excesso de crescimento (Souza \& Casali, 1986).

Nos dois anos do experimento foram obtidos bulbos das classes 3 a 6 (Tabela 1). Para obtenção de melhor preço pago ao produtor é desejável que haja maior produção de bulbos classe 6 (graúdos) que classe 3 (miúdos). Em 1999, o peso dos bulbos classificados como miúdos foi maior (Tabela 1) o que pode estar relacionado à menor produtividade total de bulbos, uma vez que foram provenientes da peneira de menor tamanho e às características climáticas indesejáveis (altas temperaturas e chuvas em excesso no período de bulbificação). Em 2000 , os alhos predominaram nas classes 4 e 5 , o que pode ter sido influenciado por condições climáticas mais favoráveis, tais como temperaturas mais baixas durante a bulbificação, fator bastante importante no desenvolvimento de bulbilhos maiores.

A cobertura morta com palha de arroz seca acarretou menores produtividades total e comercial no ano de 1999 e em 2000 (Tabela 1). Esses resultados podem ser explicados pela menor evaporação de água e maior retenção de umidade pelo solo com cobertura morta, fator que favorece o pseudoperfilhamento, concordando com os resultados obtidos por Vasconcellos et al. (1971), Carmo (1984) e Araújo et al. (1993). Não houve influência da cobertura vegetal na ocorrência de outros defeitos no alho.

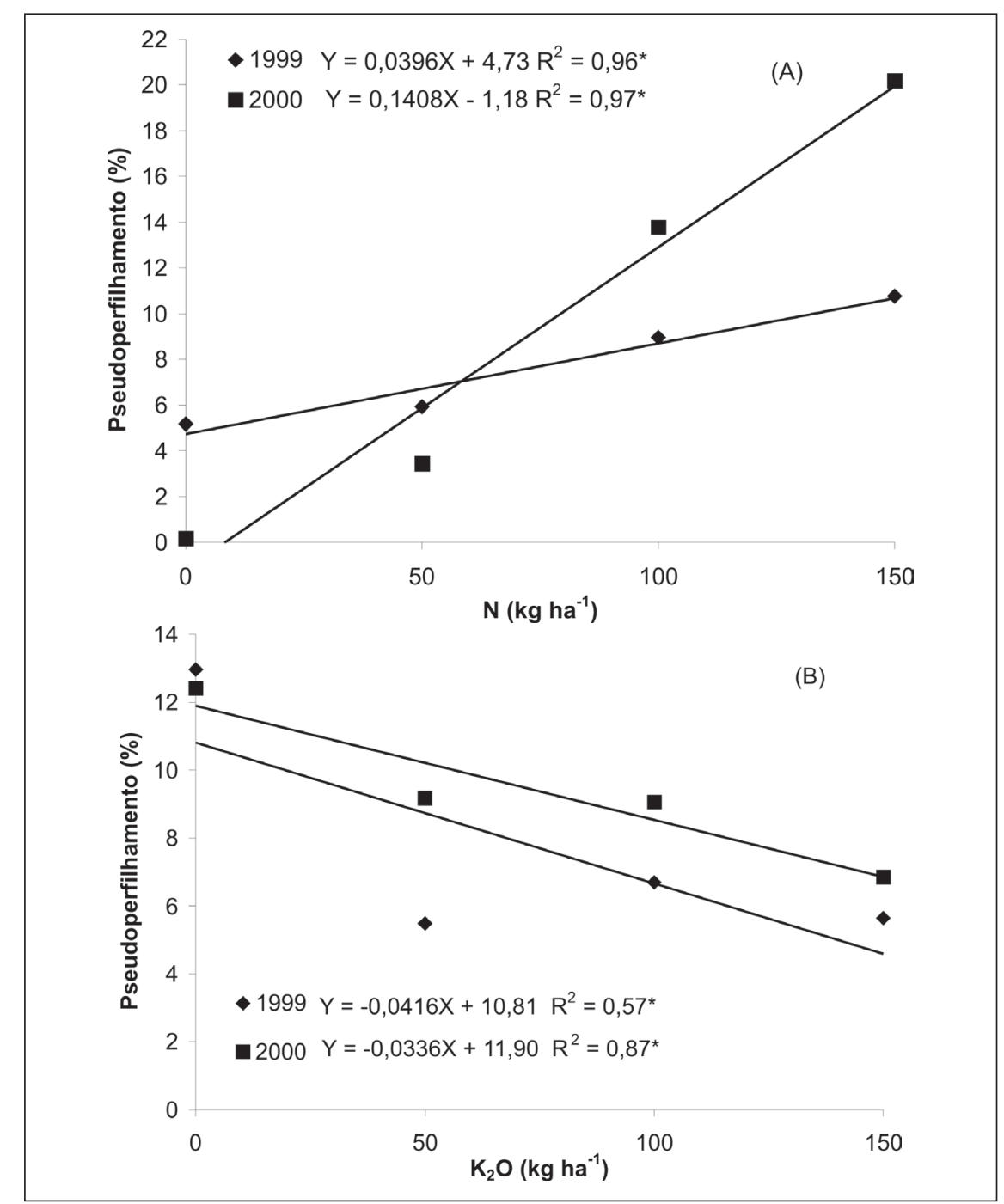

Figura 3. Ocorrência de pseudoperfilhamento no alho em função das doses de $\mathrm{N}$ (A) e $\mathrm{K}_{2} 0$ (B) $(* \mathrm{p}<0,05)\left(\right.$ Secondary bulb growth of garlic as affected by $\mathrm{N}$ rates $(\mathrm{A})$ and $\mathrm{K}_{2} \mathrm{O}$ rates $(\mathrm{B})$. $(* \mathrm{p}<0,05))$. Campinas, IAC, 1999-2000.

A adubação nitrogenada não influenciou a produtividade de alho nas classes de tamanho de bulbos em 1999, sendo que, em 2000, foram estimados os valores máximos com as doses de 53; $75 ; 81$ e $104 \mathrm{~kg} \mathrm{ha}^{-1}$ de $\mathrm{N}$ para as classes 3; 4; 5 e 6, respectivamente (Figura 1). A produtividade total alcançou valores máximos estimados de 3.922 e $8.689 \mathrm{~kg}$ ha $^{-1}$ com 74 e $107 \mathrm{~kg} \mathrm{ha}^{-1} \mathrm{~N}$ em 1999 e 2000, respectivamente (Figura 2a). A produtividade comercial $\left(3.563 \mathrm{~kg} \mathrm{ha}^{-1}\right)$ foi verificada com $68 \mathrm{~kg} \mathrm{ha}^{-1} \mathrm{~N}$ em 1999 (Figura 2a) enquanto em 2000, não houve resposta às doses de nitrogênio. $\mathrm{O}$ aumento da produtividade total de bulbos com a adubação nitrogenada também foi observado por Resende et al.
(1993), Resende et al. (2000), Alvarenga \& Santos (1982), Ferrari \& ChurataMasca (1975) e Menezes Sobrinho et al. (1974). Por outro lado, Costa et al. (1993) não verificaram efeito significativo para o nitrogênio na produtividade total e comercial do alho com $120 \mathrm{~kg} \mathrm{ha}^{-1} \mathrm{~N}$, assim como Lipinski et al. (1995) na produtividade total com $240 \mathrm{~kg} \mathrm{ha}^{-1} \mathrm{~N}$.

A menor dose de $\mathrm{N}$ obtida em relação à produtividade total para o valor máximo estimado da produtividade comercial do alho pode ser devida ao aumento do pseudoperfilhamento que foi linear com o aumento das doses de nitrogênio aplicadas em 1999 e 2000 (Figura 3a). Esses resultados concordam com Alvarenga \& Santos (1982) e 
Resende \& Souza (2001), Resende et al. (2000), Nakagawa (1993) e Silva (1991).

A adubação potássica promoveu aumento da produção comercial do alho alcançando máximo com $94,5 \mathrm{~kg} \mathrm{ha}^{-1}$ $\mathrm{K}_{2} \mathrm{O}$ em 1999. No ano seguinte, a resposta às doses de $\mathrm{K}$ foi linear (Figura 2b). O pseudoperfilhamento, por sua vez, teve redução linear com o aumento da dose de $\mathrm{K}$ no solo nos dois anos (Figura 3b), mostrando que o potássio tem relação direta com a qualidade do produto (Marschner, 1995). Os resultados obtidos são contraditórios àqueles obtidos por Büll et al. (2002), que não obtiveram resposta ao pseudoperfilhamento com as doses de $\mathrm{K}$ aplicadas. Isso mostra a importância do estudo desse distúrbio em outros tipos de solos com níveis variáveis de potássio, pois isso pode influenciar na sua dinâmica solo-planta.

\section{REFERÊNCIAS}

ALVARENGA MAR; SANTOS MLB. 1982. Efeitos de fontes e níveis de nitrogênio sobre o desenvolvimento de duas cultivares de alho (Allium sativum L.). In: CONGRESSSO BRASILEIRO DE OLERICULTURA, 22. Anais... Vitória: SOB. p. 304.

ARAÚJO RC; SOUZA RJ; SILVA AM; ALVARENGA MAR. 1993. Efeitos da cobertura morta do solo sobre a cultura do alho. Ciência e Prática 17: 228-233.

BÜLL LT; BÔAS RLV; FERNANDES DM; BERTANI RM. 2001. Fertilização potássica na cultura do alho vernalizado. Scientia Agricola 58: 157-163.

BÜLL LT; BERTANI RMA; BÔAS RLV; FERNANDES, DM. 2002. Produção de bulbos e incidência de pseudoperfilhamento na cultura do alho vernalizado em função de adubações potássicas e nitrogenadas. Bragantia 61: 247-255.

CARMO CAS. 1984. Efeitos da cobertura do solo e da freqüência de irrigação na cultura do alho (Allium sativum L.). em dois locais de altitudes diferentes do Estado do Espírito Santo. Viçosa: UFV. 61p (Tese doutorado).

CORREA TM; PALUDO SK; RESENDE FV; OLIVEIRA PSR. 2003. Adubação química e cobertura morta em alho proveniente de cultura de tecidos. Horticultura Brasileira 21: 601604.

COSTA TMP; SOUZA RJ; SILVA AM. 1993. Efeito de diferentes lâminas de água e doses de nitrogênio sobre a cultura do alho (Allium sativum L.) cv. Juréia. Ciência e Prática 7: 239-246.
EMPRESA BRASILEIRA DE PESQUISA AGROPECUÁRIA - Serviço Nacional de Levantamento e Conservação de Solos. 1999. Sistema brasileiro de classificação de solos. Brasília: Embrapa Produção de informação. 412p.

FERRARI VA; CHURATA-MASCA MGC. 1975. Efeitos de níveis crescentes de nitrogênio e bórax na produção do alho (Allium sativum $\mathrm{L}$.). Científica 3: 254-262.

KENT BT; MAY DM; GUERARD JP; RIRIE D; HATAKEDA JJ 1988. Diagnosing nutrient needs of garlic. California Agriculture 2: 2829.

KRARUP CH; TROBOK SV. 1975. Efectos de sistemas de plantación sobre rendimiento calidad del bulbo y aprovechamiento de la fertilization nitrogenada en ajo (Allium sativum L.). Fitotecnia Latinoamericana 11: 39-42.

KONKEL S; SOUZA RJ; NOGUEIRA FD. 1992. Efeitos do vinhoto e do cloreto de potássio sobre os rendimentos total e comercial e superbrotamento de bulbos de alho (Allium sativum L.) Ciência e Prática 16: 54-60.

LIPINSKI V; GAVIOLA HERAS S; FILIPPINI MF. 1995. Effect of irrigation, nitrogen fertilization and clove size on yield and quality of coloured garlic (Allium sativum L.). Ciência del Suelo 13: 80-84.

LISBÃO RS; SIQUEIRA WJ; FORNASIER JB; TRANI PE. 1993. Alho. In: FURLANI AMC; VIEGAS GP (eds). O melhoramento de plantas no Instituto Agronômico. Campinas: Instituto Agronômico. p. 222-253.

MARSCHNER H. 1995. Mineral nutrition of higher plants. New York: Academic Press. 889 p.

MENEZES SOBRINHO JA; NOVAIS RF; SANTOS HL; SANS LMA. 1974. Efeitos da adubação nitrogenada, de diferentes espaçamentos entre plantas e da cobertura morta do solo sobre a produção do alho. Revista Ceres 21: 458-469.

MORAES EG; LEAL MLS. 1986. Influência do nível e épocas de aplicação de nitrogênio na incidência do superbrotamento na cultura do alho. Horticultura Brasileira 4: 61. (Resumos).

NAKAGAWA J. 1993. Nutrição e adubação da cultura do alho. In: FERREIRA ME; CASTELANE PD; CRUZ MCP (Eds.) Nutrição e adubação de hortaliças. Piracicaba: Potafós. p. 341-380.

NOGUEIRA MCS. 1997. Estatística experimental aplicada à experimentação agronômica. Piracicaba: ESALQ-Depto Matematica e Estatistica. 250p.

OLIVEIRA CM; SOUZA RJ; MOTA JH; YURI JE; RESENDE GM. 2003. Determinação do ponto de colheita na produção de alho. Horticultura Brasileira 21: 506-509.

RAIJ B; QUAGGIO JA; CANTARELLA H; FURLANI AMC. 1997. Recomendações de adubação e calagem para o estado de São Paulo. Campinas: Instituto Agronômico/ Fundação IAC. cap.20 (Boletim n $\left.{ }^{\circ} 100\right)$.
RESENDE FV. 2004. Alho. Cultivar HF 28: 1-5 (Circular encartado).

RESENDE GM; SOUZA RJ. 2001. Doses e épocas de aplicação de nitrogênio sobre a produtividade e características comerciais de alho. Horticultura Brasileira 19: 126-129.

RESENDE GM; SOUZA RJ; LUNKES JA. 1993. Influencia do nitrogênio e paclobutrazol em alho cv. Quitéria. Horticultura Brasileira 11: 126-128.

RESENDE FV; OLIVEIRA PSR; SOUZA RJ; SOUZA RJ. 2000. Crescimento, produção e absorção de nitrogênio do alho proveniente de cultura de tecidos, cultivado com doses elevadas de nitrogênio. Horticultura Brasileira 18: 31-36.

SAS Institute INC. 1996. The SAS-system for windows: release 6.11(software). Cary: SAS Institute.

SENO S; FERNÁNDEZ FM; SASAKI JLS. 1994. Influência de doses e épocas de aplicação de nitrogênio na cultura do alho (Allium sativum L.) cv. Roxo Pérola de Caçador, na região de Ilha Solteira-SP. Cultura Agronômica 3: 9-20.

SILVA N; OLIVEIRA GD; VASCONCELOS EFC; HAAG HP. 1970. Nutrição mineral de hortaliças: XI. Absorção de nutrientes pela cultura do alho. O Solo 62: 7-17.

SILVA OG.1991. Efeitos de épocas de suspensão da irrigação e de três níveis de nitrogênio na cultura do alho (Allium sativum $L$.). Botucatu: UNESP. 77p. (Tese mestrado).

SOARES SF. 1979. Influência do tamanho do bulbilho na produção de seis cultivares de alho (Allium sativum L.). Viçosa: UFV. 56p. (Tese).

SOUZA RJ; CASALI VWD. 1986. Pseudoperfilhamento: uma anormalidade genético-fisiológica em alho. Informações Agropecuárias 12: 36-41.

SUMI S; CASTELLANE F; BELLINGIERI P; CHURATA-MASCA MGC. 1986. Cobertura morta e doses de superfostato simples na cultura do alho. Horticultura Brasileira 4: 3234.

TAVARES M; TRANI PE; SIQUEIRA WJ. 1998. Alho. In: FAHL JI; CAMARGO MBP; PIZZINATTO MA; BETTI JA; MELO AMT; DEMARIA IC; FURLANI AMC (eds.). Instruções agrícolas para as principais culturas econômicas. $6^{\text {a }}$. ed. rev. atual. Campinas: Instituto Agronômico. p. 175-176. (Boletim IAC, 200).

TRANI PE; TAVARES M; SIQUEIRA WJ; SANTOS RR; BISÃO LG; LISBÃO RS. 1997a. Cultura do alho: recomendações para seu cultivo no estado de São Paulo. Campinas: Instituto Agronômico. 39 p. (Boletim Técnico, 170).

VASCONCELLOS EFC; SCALOPI EJ; KLAR AE. 1971. Influência da irrigação e da adubação nitrogenada na precocidade e superbrotamento da cultura do alho (Allium sativum L.). O Solo 63: 15-19. 